\title{
Beyond the Boundaries: Enigma of Distinguishing Exophytic Upper Pole Renal Cell Carcinoma From an Adrenal Mass
}

\author{
Shalini T. Laroiaa, d, Vikas Jain ${ }^{\mathrm{b}}$, Archana Rastogic
}

\begin{abstract}
We report the clinical presentation, imaging findings, intra-operative appearance and pathological results of an exophytic renal cell carcinoma (RCC) of the upper pole of kidney which was presumed to arise from the ipsilateral adrenal gland. The origin of the mass was virtually indistinguishable on imaging and intraoperatively. It was determined only at histopathology examination of the resected tumor specimen. The authors are presenting few observations based on retrospective analysis of imaging and intra-operative finding of this diagnostic dilemna. This case highlights the prudent use of imaging and management approach in this group of tumors, so that adequate treatment strategies can be made preoperatively.
\end{abstract}

Keywords: Renal cell carcinoma; Adrenal mass; Magnetic resonance imaging; Laparoscopic resection

\section{Introduction}

Upper pole exophytic renal cell carcinoma (RCC) and an ipsilateral adrenal mass may be diagnostically indistinguishable by virtue of their large size and anatomical contiguity. Only few studies regarding the same are documented in literature. There are multiple differential diagnosis of adrenal pseudo masses. We have described an upper pole renal mass whose origin could not be discriminated from the ipsilateral adrenal gland on pre-operative magnetic resonance imaging (MRI) and was presumed arising from the left adrenal gland due to its radiological findings.

Manuscript accepted for publication June 23, 2015

aDepartment of Radiology, Institute of Liver and Biliary Sciences, Sector D-1, Vasant Kunj, New Delhi 110070, India

bDepartment of Renal Transplant and Urology, Institute of Liver and Biliary Sciences, Sector D-1, Vasant Kunj, New Delhi 110070, India

'Department of Pathology, Institute of Liver and Biliary Sciences, Sector D-1, Vasant Kunj, New Delhi 110070, India

${ }^{\mathrm{d} C o r r e s p o n d i n g ~ A u t h o r: ~ S h a l i n i ~ T h a p a r ~ L a r o i a, ~ D e p a r t m e n t ~ o f ~ R a d i o l o g y, ~}$ Institute of Liver and Biliary sciences, Sector D-1, Vasant Kunj, New Delhi 110070, India. Email: thaparshalini@gmail.com

doi: http://dx.doi.org/10.14740/wjnu220w
The mass (which was almost $12 \mathrm{~cm}$ in size) was resected using laparoscopic technique and was differentiated on pathological and immunohistochemical analysis of the resected specimen. In additon, a small synchronous intra-cortical RCC involving the mid-pole of the same kidney was identified only at histopathology. Retrospective analysis of the MRI and clinical investigations performed for evaluation of the tumor revealed the lacunae and markers which should always be considered during evaluation of such a mass, so as to preempt the clinician to decide the mangement and surgical strategy in similar lesions.

\section{Case Report}

A 48-year-old lady was referred to our tertiary care institute for assessment of lump in the left flank with associated intermittent dull-aching pain since 1 month. The patient was on pain-relief medication for the same. No associated symptom of hematuria, pyuria, lithuria, recurrent urinary tract infections or fever was elicited. Co-morbidities such as diabetes, hypertension and asthma were absent. No obvious weight or appetite loss was observed. She did not give history of alcohol or substance abuse. The patient was post menopausal without prior history of surgery in the past. She did not report symptoms of profuse intermittent sweating, palpitations or fluctuations in blood pressure. Physical examination revealed a globular non-tender lump at the left renal angle and left hypochondrium. It showed a well-defined lower margin, approximately 6 $\mathrm{cm}$ below left costal margin on bimanual palpation and was seen to move with respiration. Laboratory investigations including urinary vanillylmandelic acid (VMA), serum cortisol and 24-h urine metanephrine levels were all within normal reference range (Table 1). MRI of abdomen, performed to investigate the lump in the left flank, showed a large soft tissue well encapsulated mass, occupying the left flank which was not identified separately from the left adrenal gland (Fig. 1a). In addition, it was noted that the tumor had a small segment of indistinct fat planes with the upper pole of the left kidney (Fig. $1 b)$. The mass showed soft tissue heterogeneity with areas of necrosis and fibrosis appearing bright and dark, respectively, on T2WI (Fig. 1a). A small well-defined rounded lesion appearing hyperintense to the renal parenchyma was seen in the cortex of left renal mid-lower pole. Its signal was suggestive of high density contents (Fig. 1c, d). Both kidneys showed nor- 
Table 1. Laboratory Investigations

\begin{tabular}{|c|c|c|}
\hline Test performed & Results & Reference range \\
\hline Hemoglobin & 13.0 & $12-15 \mathrm{~g} / \mathrm{dL}$ \\
\hline Platelets & 160 & $150-400 \times 10^{9} / \mathrm{L}$ \\
\hline TLC & 10.1 & $4-11 \times 10^{3} / \mathrm{L}$ \\
\hline DLC & N37L50M06E07 & $\mathrm{N}: 40-75 \%, \mathrm{~L}: 20-45 \%, \mathrm{M}: 2-10 \%, \mathrm{E}: 1-6 \%$ \\
\hline Urea & 34.4 & $15-40 \mathrm{mg} / \mathrm{dL}$ \\
\hline $\mathrm{Cr}$ & 0.44 & $0.2-1 \mathrm{mg} / \mathrm{dL}$ \\
\hline $\mathrm{Na} / \mathrm{K}$ & $144.4 / 3.7$ & Na: 136 - 145, K: 3.5 - 5 \\
\hline Total bilirubin/direct bilirubin & $0.3 / 0.1$ & $0.3-1.2 \mathrm{mg} / \mathrm{dL} / 0.2 \mathrm{~g} / \mathrm{dL}$ \\
\hline AST/ALT & $21 / 22$ & 5 - 40, 7 - 35 IU/L respectively \\
\hline GGT & 8 & 7 - $64 \mathrm{IU} / \mathrm{L}$ \\
\hline Total protein/Alb & $6.7 / 3.7$ & $6-8 / 3.5-5.2 \mathrm{~g} / \mathrm{dL}$ respectively \\
\hline Urine routine & Unremarkable & \\
\hline Urine culture & Sterile & \\
\hline \multicolumn{3}{|l|}{ 24-h urinary markers } \\
\hline Metanephrines & 81.91 & $27-155$ \\
\hline Nor-metanephrines & 168.79 & $46-256$ \\
\hline VMA & 4.14 & $1.6-4.2$ \\
\hline Epinephrines & 3.57 & $1.3-10.7$ \\
\hline Nor-epinephrines & 15.36 & $8.9-61.60$ \\
\hline Dopamine & 185.18 & $40-390$ \\
\hline
\end{tabular}
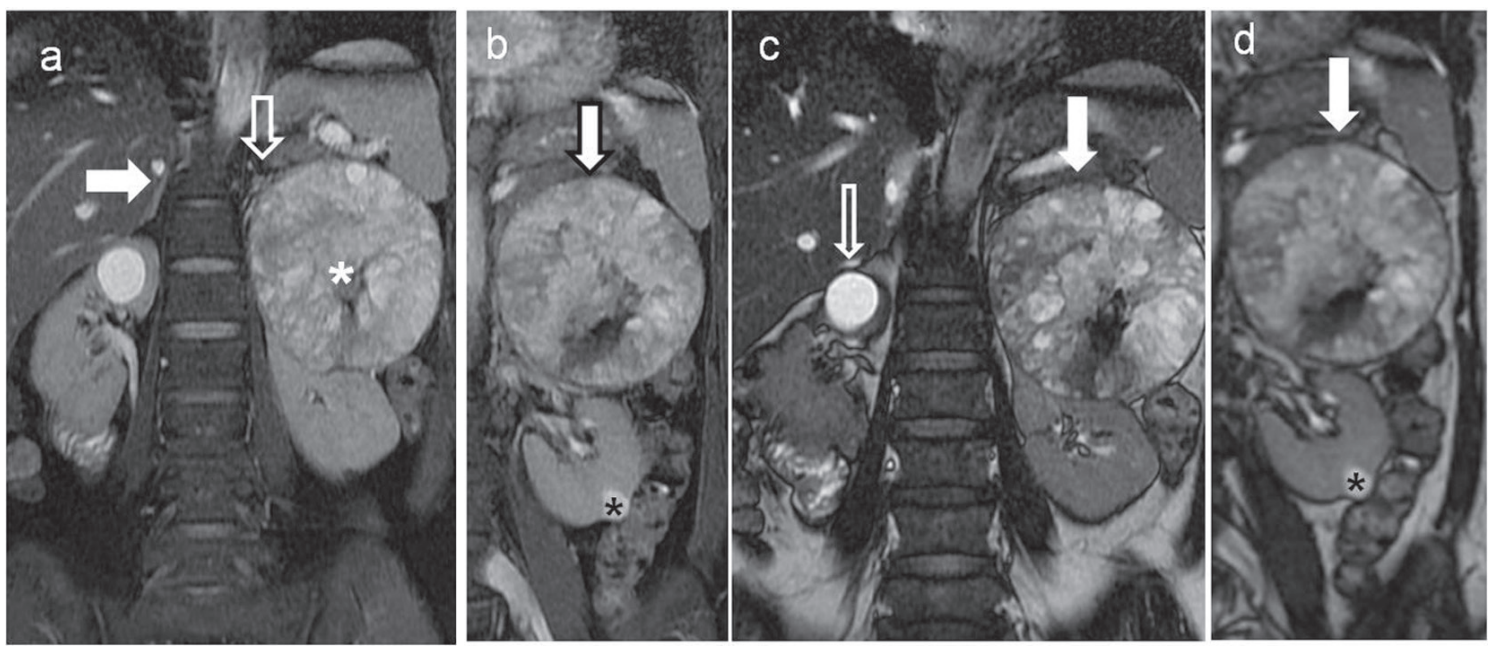

Figure 1. Non-contrast magnetic resonance imaging (MRI) of the upper abdomen showing left retroperitoneal soft tissue heterogenous renal/adrenal mass with simple cyst in the right kidney. (a) Coronal T2-weighted fat suppressed sequence of the upper abdomen showing well visualized right adrenal gland (block white arrow) and the small partially visualized left adrenal gland (white arrow) indistinct from the soft tissue mass (white asterisk) along the left renal upper pole. (b) Coronal T2-weighted fat suppressed sequence of the upper abdomen showing soft tissue mass (bold white arrow with black outline), appearing predominantly T2 hyperintense and abutting the upper left renal pole. A small T2 hyperintense lesion in the renal cortex is also seen at the lower pole of left kidney (black asterisk). (c) Coronal FIESTA sequence of the upper abdomen showing the soft tissue mass abutting the left renal upper pole (bold white arrow). In addition, a brighter well rounded simple cortical cyst is present in the right renal upper pole (bold black and white arrow). (d) Coronal FIESTA sequence of the upper abdomen showing a relatively less bright rounded lesion in the lower pole of the left kidney (asterisk) in comparison to the cortical cyst seen in right kidney in (c). 


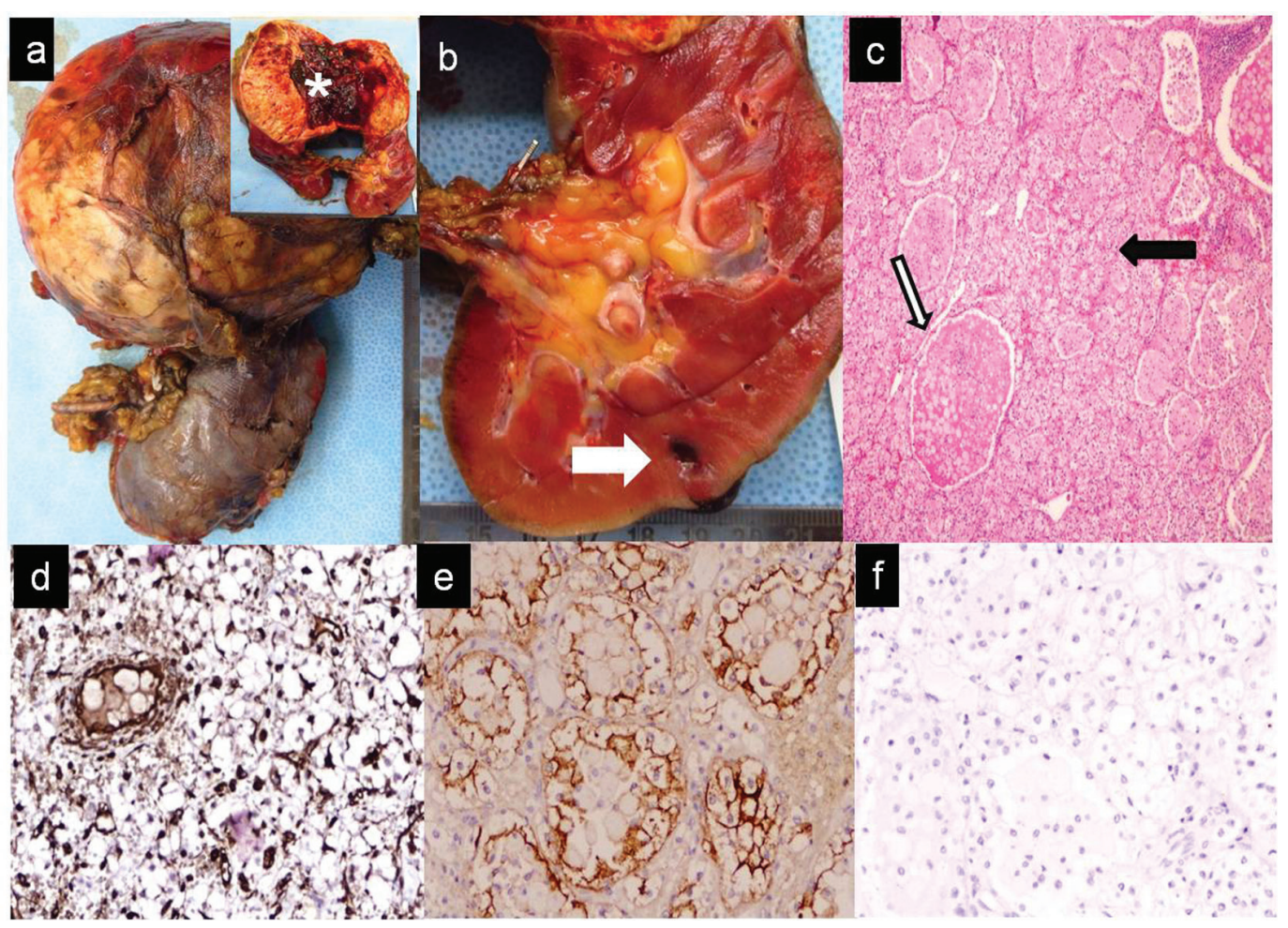

Figure 2. Gross, microscopic and immunohistochemistry findings of the soft tissue suprarenal mass removed along with radical left nephrectomy. (a) On gross pathology examination, globular tumor weighed approximately $800 \mathrm{~g}$, measured $11 \times 9 \times 8 \mathrm{~cm}$. Cut surface (inset) partially hemorrhagic, solid-cystic (white asterisk) arising from the cortico-medullary junction of the left kidney. No involvement of pelvicalyceal system, renal vessels, lymphatics or of the left ureter. (b) Small intra-parenchymal cortical lesion $(0.5 \mathrm{~cm}$ maximum dimension) in the lower pole (white bold arrow). (c) Microscopic examination of the tumor revealed a cellular tumor with nested, acinar and microcystic arrangement of malignant epithelial cells. Tumor cells displayed irregular enlarged nuclei with small nucleoli, clear cytoplasm, abundant cytoplasmic glycogen and a well-defined cell membrane (black arrow bold). These were interspersed within a highly vascularized stroma. Many glandular lumina showed presence of red blood cells (white bold arrow with black margins). (d-f) Immunohistochemistry revealed positive staining for vimentin (d), EMA (e) and negative staining for inhibin (f).

mal pelvicalyceal systems, signal intensity and parenchymal appearance on the MRI scan.

The above imaging and laboratory findings were collectively assessed and a differential diagnosis of a non-functional adrenal tumor due to the predominant suprarenal extent of the mass likely carcinoma, adrenal hemangioma, silent pheochromocytoma or metastatic deposit was considered. A possibility

Table 2. Immunohistochemistry Markers to Differentiate RCC From Adrenal Cancer

\begin{tabular}{lll}
\hline IHC markers & RCC & ACC \\
\hline EMA & + & - \\
Vimentin & + & - \\
Carbonic anhydrase-IX & + & - \\
PAX-8 & + & - \\
Calretinin & - & + \\
Inhibin & - & + \\
Melan A & - & + \\
Adrenal cortical antigens SF-1 & - & + \\
\hline
\end{tabular}

of exophytic RCC or retroperitoneal mesenchymal tumor was also considered. The adrenal was presumed to be the organ of origin. The management plan was based on the tumor size and absence of positive functional tumor markers [1].

Tumorectomy was undertaken using a left laparoscopic approach. Intra-operatively, the mass was encapsulated, vascular, without obvious infiltration into surrounding tissues, except for a small segment of the left renal upper pole. Tumor debulking with radical nephrectomy was performed (Fig. 2a). The left adrenal could not be identified separately from the mass during surgery or in the gross specimen. The final histopathological diagnosis based on microscopic appearance and immunohistochemistry markers (Table 2 ) was RCC, clear cell type, Fuhrman nuclear grade II (Fig. 2b). The postoperative course was uneventful and the patient is doing well on followup post surgery without any chemo- or radiotherapy.

\section{Discussion}

The location of the adrenal gland justifies its inclusion in the list of differential diagnosis of large exophytic upper pole renal 
tumors and retroperitoneal masses involving the supra-renal space, especially if it cannot be visually separated from the tumor bulk [2]. The logical differential for such a tumor would be an exophytic renal mass; however, careful radiological assessment of such a lesion is mandatory before surgery to obtain a pre-operative road map. A non-contrast MRI was performed for cross-sectional evaluation of our patient before surgery. Dynamic computed tomography (CT) has an advantage over MRI for "known" tumors, since it can help in diagnosis and staging in one examination. However, it has been documented that MRI is beneficial to differentiate indeterminate renal or adrenal masses compared to CT scans and to decide the management plan of such masses [3]. Soft tissue delineation of the mass as well as its fat planes, with adjacent organs is better visualized using the multiplanar ability and three-dimensional (3D) sequences on MRI. In addition specialized techniques such as diffusion, chemical shift and functional imaging with urography provide a multiparametric approach to the lesion in question [4]. It has been shown by Nakamura et al that the role of apparent diffusion coefficient of renal cancer may help in staging the tumor. These findings show that MRI may be an ideal tool to assess such masses in a wholesome manner, so as to obtain pointers to the origin of the mass pre-operatively [5]. On retrospective analysis we observed that the synchronous small tumor in the lower pole of the left kidney may have been better demonstrated and diagnosed preoperatively with a contrast-enhanced MRI, pointing towards a possibility of synchronous RCCs, i.e. an exophytic upper pole lesion and the intra-parenchymal mass. Contrast MRI would have also mapped the vascular supply of the larger tumor which could point to origin of the mass. The involvement of retroperitoneal vessels and lymph nodes could also be better delineated by a contrast study. The diagnostic challenge in this patient was essentially the inability to determine whether it was an adrenal mass with renal infiltration of a renal mass with adrenal involvement. It has been demonstrated that in cases of renal cancer, if the adrenal is not identified separately, it should also be excised during nephrectomy. On the other hand, if the adrenal gland is identified clearly on imaging, it may be spared during radical nephrectomy. The impact of adrenal sparing surgery has longterm endocrinal manifestations for the patient's wellbeing. Fluoro-deoxyglucose positron emission tomography (FDGPET) and scintigraphy may also be used for tumor origin differentiation; however, it is extremely difficult to visualize a small adrenal gland in relation to the large tumor bulk, such as seen in this patient. Resected specimens may present a diagnostic dilemma for the pathologist and the key to diagnosis lies in a combined gross, microscopic and immunohistochemical analysis.

During the course of investigations, our patient did not show positive functional markers. The size of the tumor, irrespective of its origin, mandated a surgical excision [1]. The list of our final differential diagnosis included adrenal carcinoma and an exophytic RCC as the top contenders. The management in this particular patient did not change the outcome for the patient; however, retrospective analysis of the presentation of the mass highlights factors to be considered at the time of radiological evaluation.

\section{Conclusion}

It would be prudent for the surgeon and radiologist to take the help of meticulous and recent advanced imaging techniques using dynamic MRI with newer sequences like diffusion and functional urography to attempt a detailed characterization of mass lesions in the suprarenal and renal space before a major surgery like radical nephrectomy, especially through laparoscopic technique.

\section{Acknowledgement}

Rita Gulabani, Abhishek for transcription and technical help respectively.

\section{Abbreviations}

IHC: immunohistochemistry markers; RCC: renal cell carcinoma; ACC: adrenal cortical cell carcinoma; EMA: epithelial membrane antigen

\section{References}

1. Miller BS, Doherty GM. Surgical management of adrenocortical tumours. Nat Rev Endocrinol. 2014;10(5):282292.

2. Moslemi MK, Al-Mousawi S, Dehghani Firoozabadi $\mathrm{MH}$. Renal cell carcinoma mimicking adrenal tumor. J Oncol. 2010;2010.

3. Kwon T, Jeong IG, Yoo S, Lee J, Hong S, You D, Hong $\mathrm{JH}$, et al. Role of MRI in indeterminate renal mass: diagnostic accuracy and impact on clinical decision making. Int Urol Nephrol. 2015;47(4):585-593.

4. Ramamurthy NK, Moosavi B, McInnes MD, Flood TA, Schieda N. Multiparametric MRI of solid renal masses: pearls and pitfalls. Clin Radiol. 2015;70(3):304-316.

5. Nakamura T, Yoshizako T, Araki H, Maruyama M, Uchida $\mathrm{K}$, Tamaki $\mathrm{Y}$, Ishikawa $\mathrm{N}$, et al. The relation between apparent diffusion coefficient and clinical stage of clearcell renal cell carcinoma. Clin Imaging. 2015;39(1):7275 . 